\title{
Elevation of Plasma Copeptin in Acute Myocardial Infarction in Pigs is Related to Changes in Mean Arterial Blood Pressure but not to Myocardial Ischemia
}

\author{
Anna Slagman ${ }^{1}$, Timo Jerichow ${ }^{2}$, Joachim Struck ${ }^{3}$, Jörn O. Vollert ${ }^{4}$, Dörte Mans ${ }^{1}$, \\ Malte Schröder ${ }^{1}$, Julia Searle ${ }^{1}$, Jeanette Schulz-Menger ${ }^{5}$, Ralf Schindler ${ }^{6}$, Martin \\ Möckel $^{1}$ \\ ${ }^{1}$ Department of Emergency Medicine, Charité University Medicine, Berlin, CVK and CCM, \\ Germany, \\ ${ }^{2}$ Department of Cardiology and Intensive Care, Vivantes Wenckebach-Klinikum; Berlin, Germany \\ ${ }^{3}$ Adrenomed AG, Hennigsdorf, Germany, \\ ${ }^{4}$ Clinical Diagnostics Division, BRAHMS GmbH, Hennigsdorf, Germany, \\ ${ }^{5}$ Working Group Cardiovascular MR, Department of Cardiology, HELIOS-Klinikum Berlin-Buch \\ and Charité University Medicine, Berlin, Germany; \\ ${ }^{6}$ Department of Nephrology and Intensive Care Medicine, Charité University Medicine, Berlin, CVK, \\ Germany \\ anna.slagman@charite.de
}

\begin{abstract}
Background: Copeptin increases early after myocardial infarction (MI). We used a porcine MI model with serial copeptin-testing and assessment of hemodynamic variables to evaluate the trigger for copetin-release and the liberation kinetics of copeptin in MI.
\end{abstract}

Methods: AMI was induced by percutaneous transluminal coronary angioplasty (PTCA). Blood samples were taken in 30-minute intervals.

Results: All animals $(n=4)$ had a comparable infarction size $(10.02 \pm 2.11 \%$ of the left ventricle) and elevated troponin $T$ levels. Highest copeptin levels were measured 30 minutes after the beginning of ischemia with a rapid decrease at 60 minutes, while coronary occlusion was still persisting. Animals with high copeptin values showed a significant drop of MAP (37.24\% and 54.20\% reduction from MAP-baseline).

Conclusions: In experimental AMI in four pigs, the infarction-related decrease of MAP seemed to be the trigger for the copeptin increase. Remarkably, copeptin liberation was not correlated with infarction size and copeptin values already decreased while coronary artery occlusion was ongoing.

Keywords: Copeptin, acute myocardial infarction, porcine model, hemodynamic changes, infarction size

Abbreviations: $\mathrm{AMI}=$ acute myocardial infarction; PTCA=percutaneous transluminal coronary angioplasty; $\mathrm{cMRI}=$ cardiac-magnetic resonance imaging; $\mathrm{MAP}=$ mean arterial pressure; $\mathrm{AVP}=$ Arginine vasopressin; $\mathrm{ACS}=$ acute coronary syndrome; LAD=left anterior descending artery

\section{INTRODUCTION}

Copeptin is a stable surrogate marker for Arginine Vasopressin [1] and has been investigated as an early rule out marker of acute myocardial infarction(AMI) in combination with cardiac troponin in patients with chest pain and suspected acute coronary syndrome (ACS) [2, 3]. Furthermore, in AMI copeptin levels correlate with the severity of disease and copeptin has been shown to be an independent predictor of death and heart failure [4]. Copeptin values have been reported to be increased early after onset of ischemia in patients with ST-elevation myocardial infarction (STEMI) [5] and in patients undergoing transcoronary ablation of septal hypertrophy (TASH) [6] with a fast decline to normal values. The trigger for the activation of the vasopressin system and the liberation kinetics of copeptin during spontaneous AMI remains unclear: It has been speculated that copeptin might be released (1) as part of the endocrine stress response in severe conditions such as AMI solely 
or (2) that a copeptin release might also be triggered by baroreceptor stimulation as a response on hemodynamic changes [7].

We evaluated the release kinetics of copeptin in an established porcine experimental model of AMI by serial measurements of copeptin, mean arterial blood pressure (MAP) and other vital parameters.

\section{Materials ANd Methods}

A detailed description of experimental procedures has been published previously [8]. In the original experiment 10 female pigs were investigated [9]. In the sub-experiment presented in this manuscript, data of four individuals were analyzed. Pigs were anesthetized and underwent experimental myocardial infarction by transient occlusion of the second diagonal branch of the left anterior descending coronary artery (LAD). Experimental ischemia was maintained for 60 minutes followed by reperfusion of the occluded coronary artery. Venous blood samples were taken in 30-minute intervals from four hours before until three hours after onset of ischemia. Vital parameters and oxygen saturation were continuously monitored and MAP was measured invasively through an arterial line. A catheter was placed in the urinary bladder and the quantity of expelled urine was determined in 15minute intervals. Myocardial infarct size was assessed by cardiac magnetic resonance imaging (cMRI) approximately two hours after removal of the intracoronary catheter and onset of myocardial reperfusion. Plasma copeptin was measured by a newly developed assay that was designed analog to the human copeptin assay (B.R.A.H.M.S. Thermofisher Scientific)[1]. The study protocol was approved by the state authority responsible for animal experiments LaGeSo (Landesamt für GEsundheit und Soziales, Berlin; authorization code: G0283/07) and complies with the institution guidelines, all animals received humane care and all possible steps were taken to avoid animal suffering at each stage of the experiment.

\section{RESULTS AND DisCUSSION}

Baseline troponin values were low and at the LOD of the respective troponin assay (10ng/l) in all pigs. Occlusion of the second diagonal branch of the LAD for 90 minutes caused a reproducible infarction size $(10.02 \pm 2.11 \%$ of the left ventricle) and led to elevated troponin T levels in all pigs (Figure 1). Baseline values of copeptin one hour before infarction did not differ significantly between all animals. A considerable increase of copeptin plasma levels was evident in two animals (animal no. 2 and 4), whereas two animals did not show a significant elevation of copeptin (animal no. 1 and 3). Highest copeptin levels were measured at the sampling time point 30 minutes after the onset of ischemia with a rapid decrease already 60 minutes after, while coronary occlusion was still persisting. Infarction size, heart rate or urine volume during AMI did not show any correlation with the extent of copeptin levels (data not shown). However, there was a strong negative correlation between MAP and copeptin release kinetics: animals with high copeptin values showed a significant drop of MAP (animal no. 2 and 4; 37.24\% and 54.20\% reduction from MAP-baseline value) in consequence of AMI, whereas in animals with stable MAP after coronary artery occlusion (animal no. 1 and 3;0.96\% reduction and $10.95 \%$ raise from MAP-baseline value) copeptin levels did not raise significantly (Figure 2).

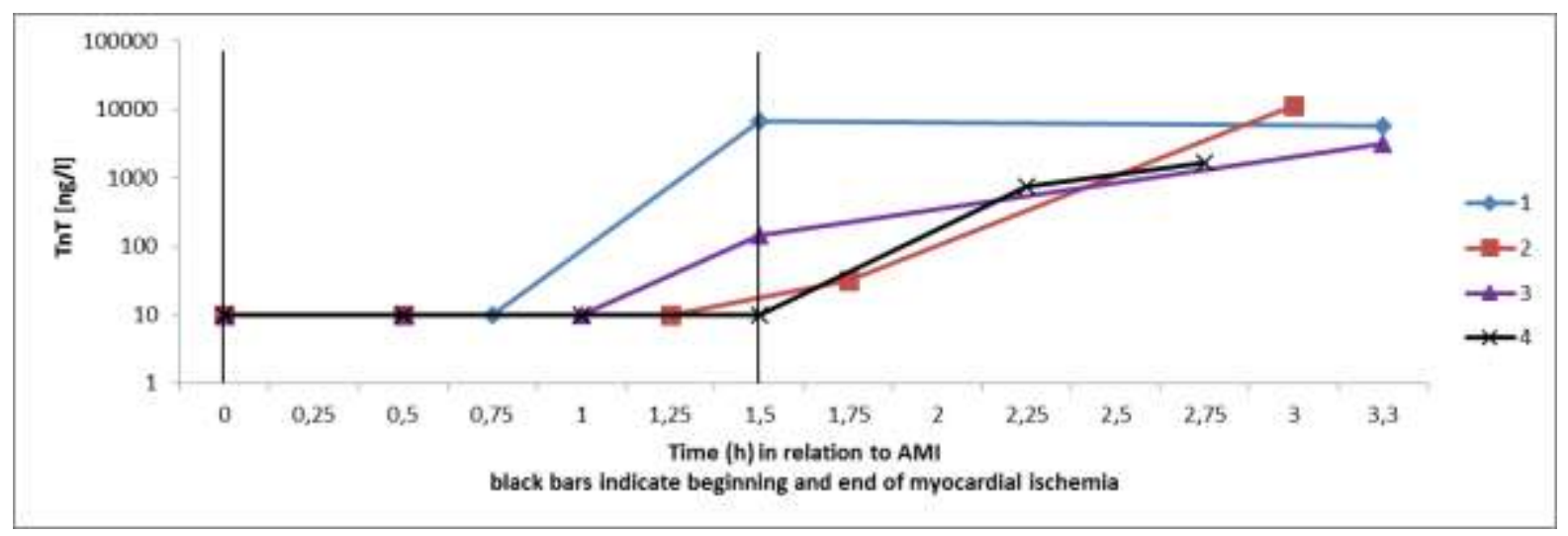

Figure1. Release pattern of cardiac troponin $T(T n T)$ 
Figure 1: Liberation kinetics of cardiac TnT in relation to time from coronary artery occlusion. In all pigs TnT increased within the first hours after induction of experimental AMI. Abbreviations: AMI acute myocardial infarction; TnT - troponin T

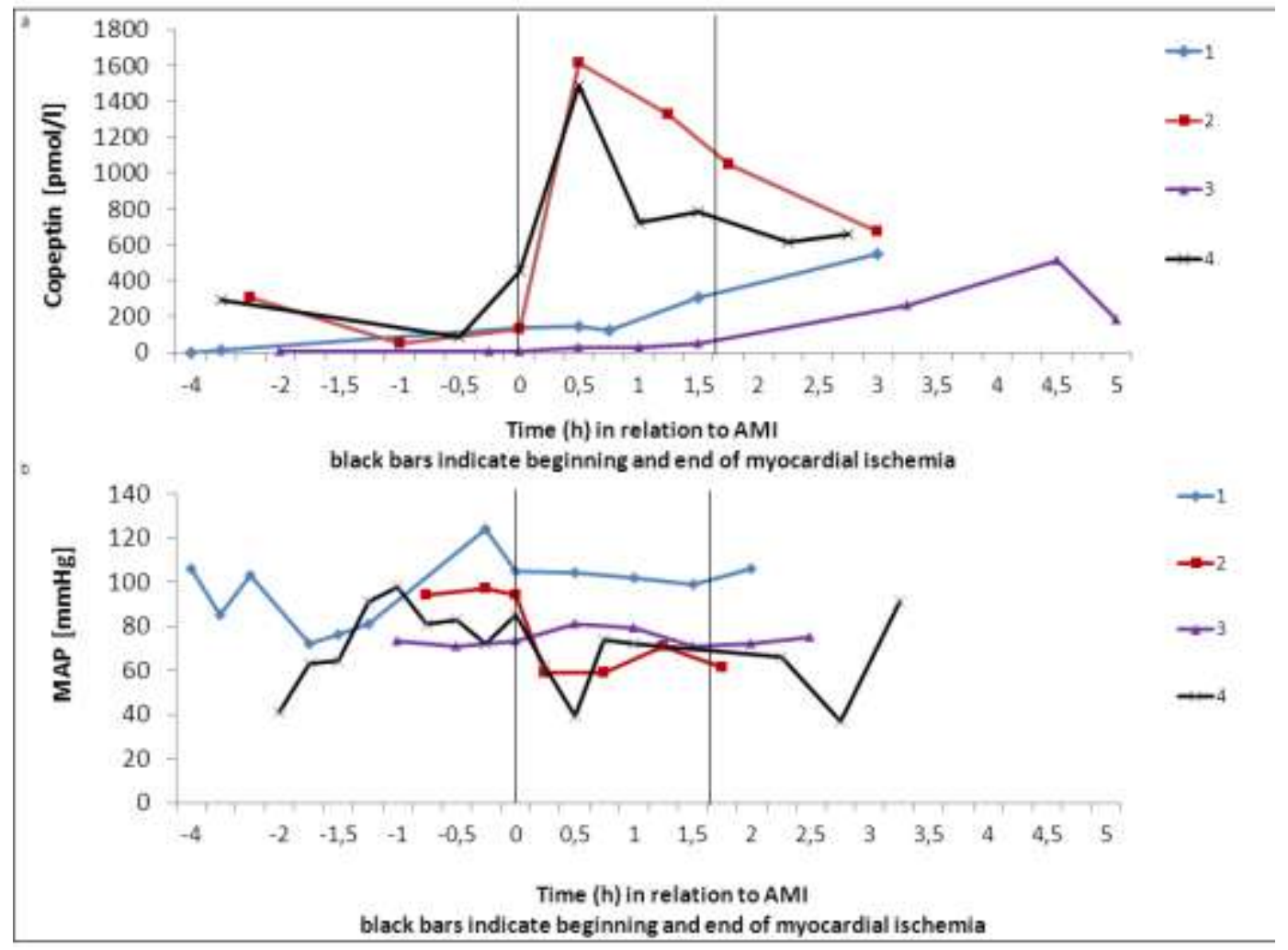

Figure2. Temporal release pattern of copeptin and corresponding values of mean arterial blood pressure $(M A P)$ in experimental AMI

Figure 2 shows the course of copeptin and mean arterial blood pressure (MAP) over time, before, during and after experimentally induced myocardial ischemia. Abbreviations: AMI - acute myocardial infarction; MAP - mean arterial blood pressure

Despite the fact that copeptin, as a surrogate marker for the activation of the vasopressin system, has become a new important biomarker with diagnostic and prognostic value in patients with ACS and heart failure $[3,10]$, the underlying mechanism for the release of copeptin subsequent to myocardial infarction and the definite liberation kinetics have sparsely been investigated until now. Activation of AVP can reflect physical stress in acute and chronic illness [11]. Results from animal models show that hypotension causes an immediate induction of AVP-gene transcription and that induced hypotension in humans leads to a significant increase in plasma vasopressin [12, 13]. An increase of plasma vasopressin was also shown in the setting of myocardial infarction due to microembolization in an ovine model [14]. In accordance with these findings, in our model of reperfused experimental AMI in pigs, the infarction-related decrease of MAP seems to be the trigger for the increase of copeptin levels. This hypothesis is supported by an experiment in monkeys: In this study, monkeys underwent experimental hemorrhage and the authors were able to show that the amount of AVP in venous blood was not related to the blood volume withdrawn but to MAP measured in an iliac artery [15]. Other authors reported that changes in plasma osmolality and hypovolemia are both closely related to AVP and copeptin levels in rats [16]. Although no continuous measurement of plasma osmolality was performed in this study, copeptin liberation due to a relevant change in plasmaosmolality or hypovolemia is very unlikely due to a standardized infusion protocol and stable diuresis in all pigs during the experiment. However, changes in osmolality cannot be excluded entirely. Remarkably, copeptin liberation was not correlated with myocardial infarct size, which did not differ significantly between all animals as assessed by cardiac-MRI. This finding is contrary to results from a study in 54 patients with ST-elevation myocardial infarction (STEMI) conducted by Reinstadler et al.: In this study copeptin correlated with early and chronic infarct size as well as maximum troponin values and thus amount of myocardial necrosis [17]. In this study, copeptin values were also inversely 
associated with left ventricular ejection fraction (LVEF), thus hemodynamic changes might also be causative for copeptin increases in patients with larger infarct size. Another explanation might be a higher extent of the endocrine stress response with increasing infarct size [11]. Hupf et al. were able to illustrate in animal experiments in isolated rat hearts that vasopressin was expressed in cardiac myocytes after acute left ventricular pressure overload [18]. Thus cardiac myocytes might also express vasopressin independently of the central mechanism in response to other stimuli such as ischemia. Regarding the liberation kinetics of copeptin, very early peak values already 30 minutes after coronary occlusion were evident while troponin $\mathrm{T}$ levels were still normal. Liebetrau et al. investigated patients undergoing transcoronary ablation of septal hypertrophy (TASH) [6]. Several blood samples before induction of myocardial ischemia and during coronary artery occlusion were drawn. Copeptin values increased significantly within 30 minutes after induction of myocardial infarction with maximum values after 60 minutes. In our study, which is limited by the small number of animals investigated, copeptin values already decreased while coronary occlusion was ongoing. This could be interpreted as an evidence that copeptin is secreted centrally and at least in the early phase is not released from the myocardium. The early increase of copeptin in patients with acute myocardial infarction makes copeptin especially useful in the early triage of patients presenting with new onset chest pain as it has been reported elsewhere [4, 19]. Based on the findings of our small series of animal experiments copeptin might not be elevated in patients with very small myocardial infarct sizes with no relevant hemodynamic changes although the threshold and prognosis remains speculative. The fact that copeptin was already decreasing while coronary artery occlusion was ongoing supports the hypothesis that the release trigger are hemodynamic changes and not myocardial ischemia. However these considerations are speculative and should be investigated in further experiments with a larger number of animals and in large cohort studies in patients with spontaneous AMI.

\section{CONCLuSiON}

In our model of experimental acute myocardial infarction (AMI) in pigs, the infarction-related decrease of mean aterial blood pressure (MAP) seemed to be a trigger for copeptin liberation. Remarkably, copeptin liberation was not correlated with infarction size and copeptin already decreased while myocardial ischemia was still persisting. Even though this is a small series of four pigs, our results are the first to show a correlation between MAP and copeptin in the setting of experimentally induced and reperfused myocardial infarction in pigs.

\section{CONFLICT OF INTEREST}

The authors are fully responsible for the content of the article. The biomarker testing (Copeptin) was funded by Thermo Fisher Scientific (Clinical Diagnostics, BRAHMS-AG, Hennigsdorf, Germany). The German Federal Ministry of Education and Research (BMBF) supported the overall project in the GO-Bio program (grant no. 0315095). The research group received grants from Thermo Fisher Scientific, Radiometer and Abbott Diagnostics. Dr. Jörn Ole Vollert is an employee of Thermo Fisher Scientific.

\section{REFERENCES}

[1] Morgenthaler, N.G., et al., Assay for the measurement of copeptin, a stable peptide derived from the precursor of vasopressin. Clin Chem, 2006. 52(1): p. 112-9.

[2] Keller, T., et al., Copeptin improves early diagnosis of acute myocardial infarction. J Am Coll Cardiol, 2010. 55(19): p. 2096-106.

[3] Reichlin, T., et al., Incremental value of copeptin for rapid rule out of acute myocardial infarction. J Am Coll Cardiol, 2009. 54(1): p. 60-8.

[4] Khan, S.Q., et al., C-terminal provasopressin (copeptin) as a novel and prognostic marker in acute myocardial infarction: Leicester Acute Myocardial Infarction Peptide (LAMP) study. Circulation, 2007. 115(16): p. 2103-10.

[5] Gu, Y.L., et al., Comparison of the temporal release pattern of copeptin with conventional biomarkers in acute myocardial infarction. Clin Res Cardiol, 2011. 100(12): p. 1069-76.

[6] Liebetrau, C., et al., Release kinetics of copeptin in patients undergoing transcoronary ablation of septal hypertrophy. Clin Chem, 2013. 59(3): p. 566-9. 
Elevation of Plasma Copeptin in Acute Myocardial Infarction in Pigs is Related to Changes in Mean Arterial Blood Pressure but not to Myocardial Ischemia

[7] Morgenthaler, N.G., Copeptin: a biomarker of cardiovascular and renal function. Congest Heart Fail, 2010. 16 Suppl 1: p. S37-44.

[8] Slagman, A.C., et al., Specific removal of C-reactive protein by apheresis in a porcine cardiac infarction model. Blood Purif, 2011. 31(1-3): p. 9-17.

[9] Sheriff, A., et al., Selective apheresis of C-reactive protein: A new therapeutic option in myocardial infarction? J Clin Apher, 2015. 30(1): p. 15-21.

[10] Narayan, H., et al., C-terminal provasopressin (copeptin) as a prognostic marker after acute non-ST elevation myocardial infarction: Leicester Acute Myocardial Infarction Peptide II (LAMP II) study. Clin Sci (Lond), 2011. 121(2): p. 79-89.

[11] Katan, M. and M. Christ-Crain, The stress hormone copeptin: a new prognostic biomarker in acute illness. Swiss Med Wkly, 2010. 140: p. w13101.

[12] Kakiya, S., et al., Role of endogenous nociceptin in the regulation of arginine vasopressin release in conscious rats. Endocrinology, 2000. 141(12): p. 4466-71.

[13] Hirsch, A.T., et al., Contribution of vasopressin to blood pressure regulation during hypovolemic hypotension in humans. J Appl Physiol, 1993. 75(5): p. 1984-8.

[14] Charles, C.J., et al., Hypothalamo-pituitary-adrenal axis response to coronary artery embolization: an ovine model of acute myocardial infarction. J Endocrinol, 1997. 152(3): p. 48993.

[15] Arnauld, E., et al., The effects of hypotension and hypovolaemia on the liberation of vasopressin during haemorrhage in the unanaesthetized monkey (Macaca mulatta). Pflugers Arch, 1977. 371(3): p. 193-200.

[16] Kawasaki, M., et al., Expression of immediate early genes and vasopressin heteronuclear RNA in the paraventricular and supraoptic nuclei of rats after acute osmotic stimulus. $\mathrm{J}$ Neuroendocrinol, 2005. 17(4): p. 227-37.

[17] Reinstadler, S.J., et al., Association of copeptin with myocardial infarct size and myocardial function after ST segment elevation myocardial infarction. Heart, 2013. 99(20): p. 1525-9.

[18] Hupf, H., et al., Evidence for a vasopressin system in the rat heart. Circ Res, 1999. 84(3): p. 36570.

[19] Mockel, M., et al., Early discharge using single cardiac troponin and copeptin testing in patients with suspected acute coronary syndrome (ACS): a randomized, controlled clinical process study. Eur Heart J, 2014.

\section{AUTHORS' BIOGRAPHY}

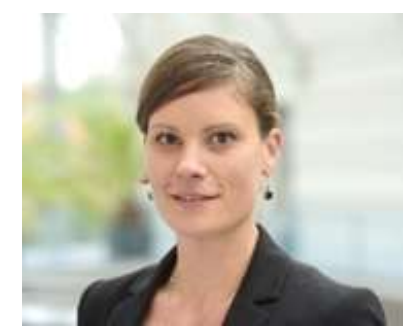

Anna Slagman (MSc), graduated in Veterinary Medicine at the Free University Berlin, Germany in 2008. She is Master of Science in Epidemiology since 2013 (Berlin School of Public Health) and works at the Charité University Medicine Berlin as a research fellow since 2008. Her key research areas are experiments and clinical studies on biomarkers in acute cardiovascular diseases and in emergency medicine. She is member of national and international societies and already published in international journals.

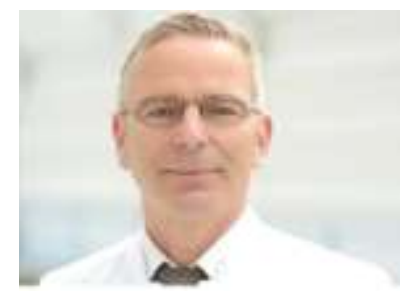

Univ.-Prof. Dr. med. Martin Möckel, graduated at the Medical School, Free University Berlin, Germany in 1991. He is Head of the Division of Emergency and Acute Medicine and Chest Pain Units of the Charité University Medicine (Campi North) in Berlin since 2010 and University Professor for Cardiovascular Process Research since 2012. Prof. Möckel is member of numerous national and international societies and has over 120 publications in international journals. 